\title{
Mitteilungen des bvU
}

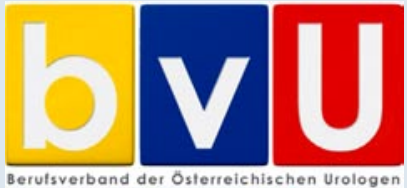

Urologe 2010 · 49:156

DOI 10.1007/s00120-009-2222-y

(c) Springer Medizin Verlag 2010

\section{Verantwortlich für den Textteil}

Dr. Karl Dorfinger

Geschäftsstelle des Berufsverbandes

der Österreichischen Urologen

Perfektastraße 28/1

A-1230 Wien

office@dorfinger.at
Höchststand erreicht. Fragt man aber Abteilungsleiter, klagen diese nach Stellenausschreibungen regelmäßig über mangelnde Bewerbungen. Die Krankenkassenreform ist ebenfalls im Vergleich zu Deutschland deutlich verzögert, wenn auch trotzdem auf geraden Schienen ins Ungewisse: Erst diese Woche wurde im Hauptverband der Österreichischen Sozialversicherungen ein Sparpaket beschlossen, dass bedingt durch die vorangegangene Regierungsvorgaben ein Einsparungsausmaß von 1,7 Mrd Euro in den nächsten 3 Jahren vorsieht. Durch Alterlimitierung wird es allein in Wien bereits im nächsten Jahr etwa 100 Kassenvertragsärzten weniger geben (derzeit gibt es knapp 2000 Vertragsärzte). Ein Blick auf die Altersverteilung der Wiener Urologen zeigt, dass von den berufstätigen Ärzten 20\% über 60 Jahre alt sind und nur $12 \%$ ein Alter von 40 Jahren oder weniger aufweisen!

Den bereits etablierten Ärzten bleibt bei einer überalterten $\mathrm{Be}$ völkerung ein zunehmender Arbeitsdruck bei sinkenden Einnahmen. Gleichzeitig steigen bürokratische und finanzielle $\mathrm{Be}$ lastungen der Ärzteschaft stetig. Obwohl es keine Vergleichsdaten gibt, weisen schon jetzt zahlreiche Experten auf zunehmende psychische Erkrankungen der Ärztinnen und Ärzte hin.

Zumindest zwei Dinge können wohl auch in Zukunft nicht folgenlos von der Politik ignoriert werden: Die stets wachsende Qualität in der medizinischen Versorgung der Menschen und die rasant voranschreitende Entwicklung neuer Diagnose- und Therapieverfahren. Es erhebt sich also die Frage, wie eine Problemlösung aussehen könnte: Wird es beispielsweise zu einer Zuwanderung ausländischer Mediziner kommen (was natürlich wieder zu einem Ärztemangel in deren Heimatländern führen würde, wie es ja jetzt schon in den osteuropäischen Ländern zu beobachten ist), oder wird, wie in
Österreich von so manchem Politiker bereits ernsthaft erwogen, das Gesundheitssystem verstaatlicht und alle Ärzte zu Billigtarifen mehr oder weniger zwangsverpflichtet (was den Nachwuchs kaum motivieren dürfte). Unter Abwägung der Vor- und Nachteile wäre es wohl das Klügste, wenn es der Politik gelänge, das Schiff wieder auf Kurs zu bringen, indem bessere Lebens- und Arbeitsbedingungen für die vorhandenen Ärzte geschaffen und somit auch mehr junge Menschen für den ärztlichen Beruf gewonnen werden. Das gebetsmühlenartig in den Medien dagegen immer wieder zitierte Argument der enormen Kostensteigerung muss - zumindest in Österreich - hinterfragt werden, denn der Anteil der Gesundheitskosten am Bruttosozialprodukt ist bei uns seit Jahrzehnten mehr oder weniger konstant.

Aus der Selektionstheorie von Charles Darwins stammt die Erkenntnis, dass in der Biologie sich stets Wege aus Krisen finden, indem Individuen sich anpassen. Das gilt wohl auch für das $\mathrm{Ge}$ sundheitssystem: Die Zahl der Ärzte wird wieder zunehmen, wenn nur der Bedarf hoch genug ansteigt. Unter den genannten Bedingungen wird ganz gemäß Darwin eine Negativselektion der Kassenärzte und im Gegenzug eine Ausweitung der Privatmedizin erfolgen. Man wird dann aber auch dafür Sorge tragen müssen, dass möglichst für Alle der Zugang zu einer menschenwürdigen Gesundheitsversorgung erhalten bleibt. Denn ein hoher Anteil unterversorgter Menschen birgt einen enormen sozialen Sprengstoff, wie man mittlerweile auch in den USA erkannt hat.

\section{Dr. Karl Dorfinger}

Facharzt für Urologie und Andrologie

Präsident des Berufsverbandes der Österreichischen Urologen 